\title{
Nomadisme chez les Touaregs
}

Hélène Claudot-Hawad

\section{(2) OpenEdition}

Journals

Édition électronique

URL : https://journals.openedition.org/encyclopedieberbere/2751

DOI : 10.4000/encyclopedieberbere.2751

ISSN : 2262-7197

\section{Éditeur}

Peeters Publishers

\section{Édition imprimée}

Date de publication : 5 octobre 2012

Pagination : 5590-5602

ISBN : 978-90-429-2718-6

ISSN : 1015-7344

\section{Référence électronique}

Hélène Claudot-Hawad, « Nomadisme chez les Touaregs », Encyclopédie berbère [En ligne], 34 | 2012, document N65, mis en ligne le 15 décembre 2020, consulté le 17 février 2022. URL : http://

journals.openedition.org/encyclopedieberbere/2751; DOI : https://doi.org/10.4000/ encyclopedieberbere. 2751

Ce document a été généré automatiquement le 17 février 2022.

(c) Tous droits réservés 


\title{
Nomadisme chez les Touaregs
}

\author{
Hélène Claudot-Hawad
}

1 Chez les Touaregs du Sahara central, le mode de vie nomade représente jusqu'à aujourd'hui une référence valorisée par rapport à la condition sédentaire même si, dans le contexte moderne, les citadins sont souvent plus riches matériellement que les nomades. Le poumon de l'économie touarègue avant la colonisation de l'Afrique était le transport caravanier qui faisait le lien entre la Méditerranée et le sud du Sahara d'une part et entre l'Atlantique et le Moyen-Orient d'autre part. En fait, les activités nomades et sédentaires étaient étroitement complémentaires, intégrées au même système économique. Le modèle confédéral touareg permettait d'appréhender comme un ensemble les groupes nomades et sédentaires dont la diversité sociale et microculturelle était considérée comme un véritable atout pour la collectivité. L'administration coloniale les a au contraire érigés en entités radicalement distinctes (dans les grilles de recensements par exemple), que ce soit sur le mode "racial», " ethnique », culturel, alors que les passages de l'état nomade à l'état sédentaire, et inversement, sont fréquents. Ce contexte a favorisé l'installation d'une frontière d'un nouveau type entre nomades et sédentaires, relevant de la représentation occidentale moderne du « bon usage » du sol et également des classifications propres à la raciologie scientifique du XIX ${ }^{\mathrm{e}}$ siècle (Blancs / Noirs).

2 Le nomadisme est au Sahara une réalité à plusieurs dimensions. Sur le plan économique, il s'agit d'un modèle de gestion des ressources, adapté à l'écologie fragile des milieux désertiques où vivent les Touaregs. Il est associé à une économie des transports qui impliquent des compétences à la mobilité sur des espaces immenses et dans un milieu extrême. Il correspond à un mode de vie particulier qui nécessite des techniques et un équipement matériel appropriés pour faciliter les déplacements. Il sous-entend également des savoirs spécifiques (géographiques, climatiques, botaniques, zoologiques...), le sens de l'orientation, et la maîtrise de plusieurs langues et de diverses cultures; en somme l'aptitude à créer des liens sociaux avec les espaces lointains pour constituer le capital humain du nomade, que l'on appellerait aujourd'hui un « réseau international ». 
3 Associé à d'autres traits de l'organisation sociale, le nomadisme chez les Touaregs définit également une position sociale privilégiée par rapport à celle du sédentaire. Enfin, il est lié à des représentations originales du monde dont les éléments constitutifs, des plus concrets aux plus abstraits, sont pensés en mouvement (cf. Claudot-Hawad 2006). Cette imagination dynamique de l'univers s'exprime à divers niveaux, dans les productions littéraires, les mythes, la cosmogonie*, les règles de l'ordre social, les conceptions psychologiques, les savoirs médicinaux, les codes esthétiques...

\section{Activités économiques nomades}

4 Jusqu'à la conquête coloniale, le contrôle des échanges transsahariens revêt une importance capitale dans l'économie saharienne et représente une source fréquente de conflits. A la fin du XIX ${ }^{\mathrm{e}}$ siècle, les principaux ports caravaniers des Touaregs sont Ouargla* et In Salah au nord, Gabès, Sfax et Tripoli au nord-est, Zawilah, Awjila, Mourzouk, Jerboub, Siwa* à l'est, Kano et Sokoto au sud, Chinguetti et les oasis du Tafilalet* à l'ouest. Par ces axes, le Sahara était relié aux marchés de la Méditerranée, de l'Orient et de l'Afrique subsaharienne. Les grandes caravanes qu'organisaient les Touaregs ont été d'abord contrôlées par le pouvoir colonial, restreintes, puis définitivement interdites avec l'instauration des nouvelles frontières étatiques. Seule subsiste, bien que de taille réduite, la caravane de sel (appelée en touareg taghlamt à l'est et azalay à l'ouest) fondée sur l'échange des dattes contre le sel que les Kel Aïr ramènent de Bilma et de Fachi, tandis qu'à l'ouest, les Touaregs de la Tademekkat se fournissent à Taoudenni. Le sel gemme est ensuite échangé au sud du Sahara contre des céréales, le mil essentiellement (cf. notamment Bernus 1972 et 1981, Gast 1968, Nicolaisen 1962, Spittler 2002).

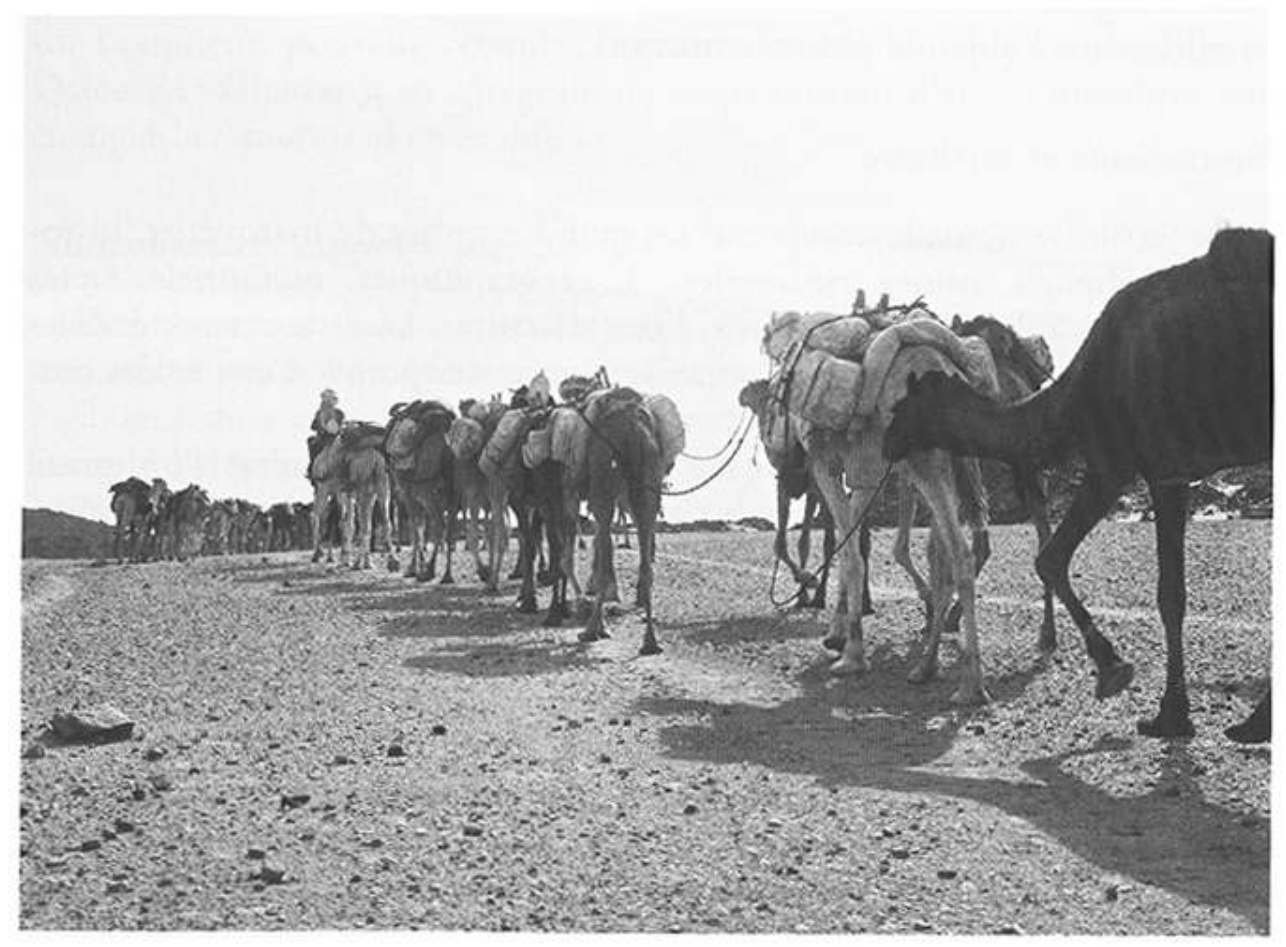

Photo 1. CARAVANe de SEL, MARS 1970

Cliché M. Gast. 

études pendant et après la période coloniale. Due soit à l'administration, soit à des géographes, ethnologues, botanistes, la majorité de ces travaux s'insère dans des perspectives de développement, certaines liées à la question des sécheresses (Copans 1975, Spittler 1993, Sircoulon 1985) et d'autres à la volonté politique de sédentariser les nomades. Des analyses détaillées concernent les techniques d'élevage, la diversification des troupeaux, l'usage alterné des pâturages, les déplacements en fonction des saisons, les mouvements pendulaires annuels, le croisement des parcours nomades (Ag Foni 1979, Ag Sidiyene 1996, Barral 1967 et 1974, Bernus 1974, 1979 et 1981, Blanc-Pamard \& Boutrais 1994, Bonte 1971 et 1973, Barrau, Bonte, Digard \& al. 1973, Copans 1975, Gallais 1975, Galloy 1958, Nicolas 1947, Vallet 1960 et 1975). Le travail astreignant de gardiennage a été parfois finement observé (Spittler 2003). Les activités complémentaires de subsistance comme la chasse et la cueillette ont également été étudiées (Bernus 1967 et 1999, Gast 2000).

La valorisation du nomadisme pastoral et celle du grand bétail, camelin mais aussi bovin, hiérarchisent également les valeurs accordées à l'alimentation lactée (Gast 1968) et aux vertus des divers types de lait. La consommation du lait de chamelle par exemple est jugée indispensable à la santé et à la beauté du corps.

7 Si la rationalité de la gestion nomade des ressources pastorales a été souvent soulignée, la notion de «territoire » d'un point de vue nomade a fait parallèlement l'objet de plusieurs travaux.

\section{Nomadisme et territoire}

8 Le territoire nomade comprend un grand nombre de marqueurs, historiques (tifinagh, ruines médiévales...), géographiques, mémoriels, sacrés (tombes des martyrs et des saints, lieux de culte). Dans les cartes établies par les nomades, le territoire s'organise autour des points d'eau et des sentiers qui les relient (Bernus 1982 et 1988).

Le territoire est considéré par les Touaregs comme le résultat d'un travail qui seul permet de rendre viable la nature à l'état brut (Claudot-Hawad 1986 et 2008). La terre en effet ne protège que si elle est parcourue, domestiquée, modelée par les itinéraires nomades qui régulent les relations entre les êtres humains et le désert ou, dit autrement, entre la culture et la nature. Cette image renvoie à l'usage économique raisonné du sol, géré et ordonné de manière à rendre optimales l'exploitation et la reproduction des ressources. Elle correspond également à des usages sociaux et symboliques $\mathrm{du}$ territoire auquel s'identifient les individus et les groupes. Concrètement, toute unité sociale, de la plus petite (le campement) à la plus grande (la société tout entière), est associée à un territoire-parcours extensible selon les saisons, croisant d'autres parcours qui dessinent les trames complexes du vaste maillage territorial et politique touareg. Chaque groupe exerce des droits d'usage prioritaires bien que non exclusifs sur son parcours. Ces prérogatives se déterminent donc par rapport aux mouvements dans l'espace définis par des étapes coutumières, liés à des droits territoriaux définis, mais aux contours flexibles et négociables suivant les conditions climatiques ou politiques.

Les ressources en jeu sont essentiellement les pâturages, les points d'eau naturels ou aménagés (sources, mares, puisards, guelta, puits), le gibier, les produits de cueillette et 
le bois. Dans l'organisation touarègue, ces biens ne peuvent être appropriés individuellement. Leur contrôle s'établit aux différents échelons de la communauté, représentés autrefois par des chefs-arbitres qui endossaient la responsabilité de la gestion du territoire par rapport aux groupements voisins et aux instances plus larges (au niveau confédéral et interconféderal). Les espaces attribues aux différents groupes sont vus comme un emboîtement de territoires, chacun articulé à la manière d'un « corps » (taghasa) et représentant, dans la figure supérieure, une partie du tout.

11 Dans cette perspective, le tracé des itinéraires nomades traduit la mise en dialogue des deux faces du monde jugées indispensables l'une à l'autre, c'est-a-dire la nature sauvage et l'espace domestiqué, ou encore le désert et la tente, l'inconnu et le connu, l'altérité et l'identité, le masculin et le féminin... De même, les routes-frontières ont un rôle fondamental d'interface, lieu de négociation des relations qui permettent de transformer des ennemis potentiels en partenaires.

Cette manière d'envisager les limites, en leur prêtant un caractère réversible (d'opposition ou de lien), s'intègre à une gestion de l'espace ouverte sur l'extérieur, pouvant s'étendre horizontalement (en «rhizome » diraient Deleuze et Guattari), en agrégeant au corps existant d'autres membres sans changer la structure d'ensemble.

\section{Nomadisme et rapports sociaux}

13 Etre nomade au Sahara central signifie pratiquer une mobilité pourvoyeuse non seulement de biens économiques (incluant l'entretien des ressources écologiques,) mais aussi créatrice de liens sociaux et symboliques, mouvement assimile a des valeurs hautement positives. Le nomadisme, perçu comme le contraire de l'errance, suppose des déplacements spatiaux organisés et régulés qui "bâtissent" et remodèlent à chaque déplacement le corps politique et territorial. Dans ce système, l'importance sociale d'un individu ou d'un groupe se mesure à l'amplitude de sa mobilité et des étapes parcourues. Se déplacer sur des distances lointaines, faire le lien avec des mondes voisins ou extérieurs, signifie être capable de mobiliser un réseau social vaste et donc être puissant. Au contraire, le cantonnement à des espaces restreints manifeste l'indigence des relais et des alliés, et le manque de compétences (politiques, économiques, linguistiques, culturelles) pour créer des relations avec des espaces sociaux autres que ceux de la proximité immédiate. C'est pourquoi, dans ce schéma, l'autochtonie est un trait définitoire accolé aux "pauvres«, aux protégés, aux tributaires. L'élite au contraire se définit comme provenant nécessairement d'un "ailleurs" prestigieux, originaire d'un pôle d'échanges réputé où convergent à un moment donné les flux commerciaux, socio-politiques et culturels les plus importants. Les mythes d'origine des groupements touaregs portent la marque des remaniements opérés en fonction du déclin, de l'abandon ou de l'émergence de ces pôles d'attraction.

14 Cette organisation valorise ainsi le caractère composite et flexible de la trame sociale et le rôle essentiel des interfaces, qu'elles soient incarnées par des personnages médiateurs ou par des lieux carrefours. La frontière nomade ne représente pas une barrière étanche qui interdirait aux étrangers l'accès aux ressources ou encore le passage (les droits sur le sol sont prioritaires, mais non privatifs). Elle est au contraire une ligne de jonction, négociable et fluide. C'est en effet sur les frontières territoriales nomades que sont installés les puits, les marchés, les routes, les espaces sacrés ou les ancrages urbains intégrés autrefois à un système territorial aux ramifications très 
larges. Dans ce contexte, dépasser la frontière - territoriale, mais également sociale, culturelle ou linguistique - est perçu comme une source d'élargissement de soi et d'ascension sociale. Cette image caractérise la mobilité sociale chez les Touaregs et les procédés qui rendent possible le passage d'une catégorie sociale à une autre.

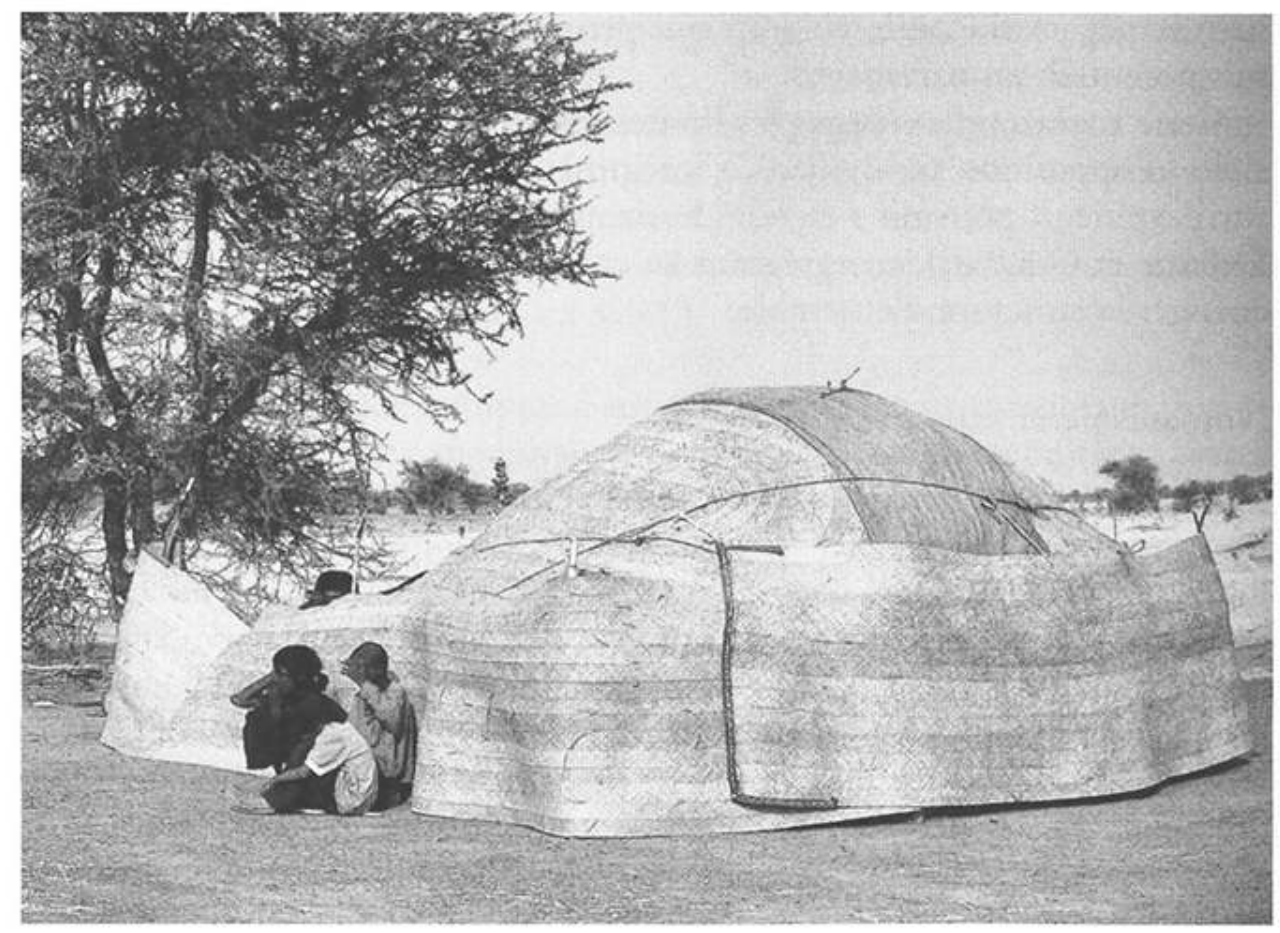

Photo 2. TENTE EN NATTES DE L'AÏR

Cliché H. Claudot-Hawad.

\section{Un modèle à penser le monde}

Le nomadisme chez les Touaregs n'est pas seulement un mode de vie, une économie ou une pratique de gestion extensive des ressources. Il représente également une philosophie, une manière de voir les réalités et d'agir sur elles. Il propose un modèle sous-jacent de l'ordre du monde qui se fonde sur l'idée que toute entité possède un caractère composite, comme le corps formé de membres différents. Dans cette vision du monde, la tension entre éléments distincts mais équivalents est nécessaire pour stimuler le système et le rendre dynamique; enfin les relations entre les parties doivent être constamment négociées et rééquilibrées pour les assembler harmonieusement.

Cette position implique un dispositif de savoirs et de pratiques mis en œuvre pour préserver d'une manière ou d'une autre la bonne « marche » du monde, c'est-à-dire a la fois sa continuité et sa croissance. Elle repose sur la capacité à produire et à gérer des relations durables entre des éléments distincts en transformation constante.

Dans l'imaginaire touareg, l'organisation de l'univers et de chaque partie qui le compose est assimilée symboliquement à la charpente d'une tente ou à l'anatomie du corps humain (Claudot-Hawad 1990). Ce parallèle renvoie à plusieurs principes. D'une part, il exprime une vision organique de la société, perçue comme une construction articulant des éléments différents mais complémentaires. D'autre part, il met en avant 
la fonction de protection de cet assemblage, soit parce qu'il constitue un abri semblable au velum de la tente, soit parce qu'il permet la motricité et l'autonomie du corps. Le troisième principe a l'œuvre est le caractère dynamique attribué à toute chose, tout élément, tout être, perçus en mouvement sur un parcours cyclique universel, semblable à celui de l'univers. Plutôt que les parties du tout qui sont en mutation constante, cette perspective met en valeur les liens qui les unissent: c'est pourquoi les rôles de médiateurs, personnages de «l'entre-deux » (ger etesnat) incarnés dans les institutions anciennes par diverses figures sociales (chef-arbitre, religieux, affranchi...) ${ }^{1}$ ou encore la fonction des lieux de rencontre et de jonction placés à l'intersection des territoires (routes, parcours, puits, marchés, villes...), sont si importants dans cette construction.

Cette structure s'applique à toute formation sociale et territoriale, quelle que soit son extension. L'homologie de nature postulée entre la société, le territoire, la maison et le corps, permet de passer d'un registre métaphorique a l'autre. Le vocabulaire politique rend compte des transferts sémantiques établis entre ces domaines : ainsi, les entités politiques et sociales sont désignées par des noms empruntés à l'anatomie humaine (" poignet », « cuisse », « hanches », " poitrine »...). De même les noms propres de nombreuses confédérations de tribus appartiennent au vocabulaire de la tente et de ses espaces intérieurs, auxquels correspondent également les diverses parties du territoire. La «mise en corps » du monde environnant, inculquée et assimilée dès l'enfance, fonctionne comme un outil de décodage de réalités d'ordre divers et un mode de construction de soi et des relations à autrui.

\section{L'art d'être nomade}

Parmi les savoirs enseignés, l'éducation touarègue met l'accent sur toutes les compétences qui permettent la mobilité, la communication et l'adaptabilité à des situations inédites. L'excellence dans l'éducation d'un Touareg se fonde sur le cumul et la synthèse de savoirs pluriels. Elle se construit comme une position généalogique prestigieuse : être de «bonne naissance » dans ce cadre signifie en effet cumuler les attaches parentales à différents lignages, plutôt qu'à un seul (Walentowitz 2003, p. 225-230). De même, apprendre correspond moins à l'acquisition d'une «tradition " par définition orthodoxe qu'à la recherche d'ouvertures sur l'extérieur. La quête de connaissance, comme le montrent divers itinéraires individuels - voir par exemple le parcours de personnalités politiques comme Kawsan* qui a dirigé en 1916 le soulèvement général des Touaregs contre la colonisation ou Mohamed Ali ag Attaher Insar (1990) - se nourrit d'expériences les plus diversifiées possibles, avec l'idée cependant que les savoirs extérieurs ne sont profitables qu'à la condition de pouvoir les trier et les contenir (eseluji) pour les recycler en une synthèse originale, obtenue grâce aux savoirs de l'intérieur de la société. Cette position à la fois ouverte et critique par rapport à la connaissance s'illustre de façon multiple (voir par exemple le récit de Welet Halatine 1998, p. 30).

On comprend dans ce contexte la valorisation du tikruru, figure du métis culturel qui maîtrise les codes de plusieurs mondes, sans être aliéné à aucun. L'éducation des enfants chez les Touaregs nomades montre à quel point l'acquisition de savoirs variés appartenant à divers horizons socioculturels est valorisée. Jusqu'aux années 1970, par exemple, les garçons des milieux nobles de l'Air faisaient des séjours d'une ou plusieurs années chez des alliés ou des clients de leurs parents, s'initiant à d'autres langues et à 
d'autres modes de vie. C'est pourquoi, les hommes - dont le rôle social est d'assurer les relations avec l'extérieur - sont polyglottes, sans compter les langues de transmission écrites en usage dans les milieux savants comme le persan, l'araméen, l'hébreu... Ainsi, les hommes touaregs de l'Air, en plus de leur langue maternelle, pratiquent couramment le haoussa, certains l'arabe, le songhay, le peul, le kanouri et le toubou. De manière générale, les Touaregs de l'Ajjer et de l'Ahaggar parlent également l'arabe et le songhay (langue des oasis), et les Touaregs de l'Ouest le songhay, et souvent le peul, l'arabe (hassanya), le dogon. Les langues coloniales, certaines devenues "nationales" et enseignées à l'école, ne sont connues par contre que d'un petit nombre, les Touaregs ayant massivement refusé l'école pendant la colonisation et s'étant ensuite heurté aux administrations nationales pour accéder à l'institution scolaire. Il n'y a guère qu'en Libye dans les années 1970-2000 qu'un nombre significatif de Touaregs a pu faire des études supérieures.

La formation au voyage et à la communication interculturelle est ainsi l'une des facettes originales de l'instruction nomade touarègue (Claudot-Hawad 2002a). Elle permet de tisser les liens qui «bâtissent l'abri », dans tous les sens du terme. L'idée de la charpente protectrice - construite par les parcours matériels ou spirituels des individus - structure fortement les rapports à la société, à la parenté et bien sûr au territoire nomade.



Photo 3. TENTE des INVITÉs 1964 (AHAggAR).

Cliché M. Gast

\section{Le nomadisme marginalisé}

Les Touaregs relèvent aujourd'hui de cinq Etats différents créés dans les années 1960 à l'issue du processus de "décolonisation »: la Libye, l'Algérie, le Niger, le Mali et le 
Burkina Faso (ancienne Haute-Volta). Ces formations politiques récentes, construites sur le modèle occidental de l'Etat-nation, ont produit des territorialités d'un nouveau type. Dans chacun de ces espaces étatiques, les zones désertiques fréquentées par les nomades occupent la périphérie. Toutes se trouvent situées à des centaines, voire à des milliers, de kilomètres des nouveaux centres de pouvoir, aux confins les plus reculés des capitales modernes, qu'elles soient méditerranéennes comme Tripoli et Alger, ou sahéliennes comme Bamako, Niamey et Ouagadougou. C'est pourquoi les Etats modernes apparaissent au Sahara comme de véritables "fabriques à minorités", reléguées aux marges des nouvelles centralités - sédentaires et urbaines - et par ailleurs séparées de leurs anciens pôles d'attraction.

Un premier constat se dégage : en moins de cinq décennies, le mode de vie nomade a drastiquement régressé chez les Touaregs. Il a quasiment disparu en Algérie et en Libye, où une politique systématique de sédentarisation a été menée dans les années 1970-1980. Il est en forte dégradation et diminution dans les Etats sahéliens, où les nomades étaient pourtant, dans les années 1960, les plus nombreux et les plus riches parmi les Touaregs et plus largement parmi les populations sahariennes. Ce recul est directement induit par les graves crises qui ont affecté et affectent encore ces régions : les unes, souvent évoquées comme une fatalité naturelle, sont climatiques et écologiques (sécheresse de 1974 et de 1984), mais elles sont étroitement liées aux autres, politiques et militaires, autant qu'économiques et sociales. Car les terres touarègues nomades représentent aujourd'hui un enjeu majeur de l'économie mondiale : leur sous-sol recèle des ressources minérales très importantes (pétrole, gaz, uranium...) qui suscitent la convoitise et la rivalité des grandes puissances économiques internationales, provoquant des luttes souterraines qui prennent diverses formes au niveau local et ont abouti à une déstabilisation totale de la région. L'interaction de ces facteurs a mis en danger les Touaregs, précipitant dans la misère et dans l'exil des milliers de familles qui ont trouvé refuge tantôt en franchissant la frontière vers d'autres régions touarègues, tantôt dans des lieux étrangers (grandes villes subsahariennes, Arabie Saoudite, etc.).

En apparence la vie nomade semble avoir peu changé. Dans les campements, le quotidien est rythmé par les tâches pastorales. Le déménagement régulier des tentes se poursuit bien que dans un rayon d'action limité (passant de 800 ou 900 kilomètres par an à moins de 100 kilomètres). D'autre part, le petit bétail a supplanté les troupeaux camelin ou bovin chez les familles désargentées. Les tâches d'élevage, autrefois confiées en partie aux "pauvres » et aux jeunes, sont prises en charge aujourd'hui par tous les membres de la société. Les caravanes sont devenues une entreprise de type individuel et non plus collectif.

Mais au-delà de ces transformations de surface, un changement de fond radical compromet l'existence même du nomadisme, qui ne peut plus subsister localement à cause de la dépossession territoriale des habitants du désert et de la fragmentation de leur espace. Aucun droit sur le sol et sur les ressources n'est reconnu juridiquement ni aux Touaregs en particulier, ni aux nomades en général. Aucune des instances anciennes d'arbitrage et de gestion ne peut s'exercer légitimement. Aucune construction du territoire ne peut s'étendre au-delà des frontières et des micro-Etats qu'elles délimitent. Dans ce contexte, même les projets d'aménagement pastoral promus par les ONG, loin de soutenir le mode de vie nomade, précipitent sa disparition, 
en ne pensant le développement qu'à partir de petites niches écologiques déconnectées de l'ensemble.

Le cadre virtuel de la mobilité nomade s'est ainsi non seulement rétréci, il s'est figé et ne permet plus d'être "nomade» au sens plein du terme. Il prépare les révoltes prévisibles des nomades sans terres de demain, spoliés de leur territoire, de leurs biens, de leurs moyens d'existence, privés de droits politiques, économiques, sociaux et culturels et amputés de l'espoir d'un avenir décent.

\section{BIBLIOGRAPHIE}

AG ATTAHER INSAR M. A., 1990 - « La scolarisation moderne comme stratégie de résistance », REMMM, $\mathrm{n}^{\circ}$ 57, p. 91-97.

AG BOUYA A., 1984 - Les conséquences de la sécheresse sur le nomadisme, Bamako, Mémoire de l'ENA.

AG FONI, E., 1979 - L'impact socio-économique de la sécheresse dans le cercle de Kidal de 1974 à 1976,

Bremen, Borda, $154 \mathrm{p}$.

AG MAHMOUD, H. 1968 - Le problème de la sédentarisation des populations nomades du Mali, Bamako, Mémoire de l'ENS.

AG SIDIYENE I., 1996 - Des arbres et des arbustes spontanés de l'Adrar des Iforas (Mali), Etude ethnolinguistique et ethnobotanique, Paris, ORSTOM/CIRAD, 138 p.

BARRAL H., 1974 - « Mobilité et cloisonnement chez les éleveurs du nord de la Haute Volta, p. les zones dites “d'endodromie pastorale » ». Cahiers de l'ORSTOM, Paris, Série Sciences Humaines, XI (2), p. 127-135.

BARRAL H., 1967 - « Les populations d'éleveurs et les problèmes pastoraux dans le nord-est de la Haute-Volta (1963-1964) », Cahiers de l'ORSTOM, Paris, Série Sciences Humaines, 4, p. 3-30.

BARRAU J., BONTE P., DIGARD J.-P. et al., 1973 - Etudes sur les sociétés de pasteurs nomades, Sur l'organisation technique et économique, Paris, Les Cahiers du Centre d'études et de recherches marxistes, 109.

BARRERE G., 1955 - La transhumance chez les Touaregs, Cannes, L'imprimerie à l'école, 24 p.

BARRIERE C. \& BARRIERE O., 1995 - Le foncier - environnement, pour une gestion viable des ressources renouvelables au Sahel, approche interdisciplinaire dans le delta intérieur du Niger (Mali), Paris, ORSTOM-CNRS.

BENOIT M., 1977 - Introduction à la géographie des aires pastorales soudaniennes de Haute-Volta, Paris, ORSTOM, 95 p.

BERNARD A. \& LACROIX, 1906 - Évolution du nomadisme en Algérie, Annuaire de Géographie, Alger/

Paris, Jourdan/Challamel, $343 \mathrm{p}$.

BERNARD A., 1906 - « Les diverses catégories de nomades », B.S. G. et Arch., Oran, janvier. 
BERNUS E., 1967 - «Cueillette et exploitation des ressources spontanées du sahel nigérien par les Kel Tamasheq », Cahiers ORSTOM, Paris, ORSTOM, 4, Série Sciences Humaines, p. 31-52.

BERNUS E. et S., 1972 - Du sel et des dattes. Introduction à l'étude de la communauté d'ln Gall et de Tegidda-n-Tesemt. Niamey, Études Nigériennes $n^{\circ} 31,128$ p.

BERNUS E., 1974 - Les Illabakan (Niger). Une tribu sahélienne et son aire de nomadisation, Atlas des structures agraires $\mathrm{n}^{\circ}$ 10, ORSTOM, Mouton, 14 cartes h.t., $116 \mathrm{p}$.

BERNUS E., 1975 - « Les composantes géographiques et sociales des types d'élevage en milieu touareg ", in Monod (T.) (éd.), Les sociétés pastorales en Afrique, tradition et développement, Londres, Oxford University Press, p. 229-244.

BERNUS E., 1977 - «L Les tactiques des éleveurs face à la sécheresse, p. le cas du sud-ouest de l'Aïr (Niger) » in Gallais (J.) (éd.), Stratégies pastorales et agricoles des Sahéliens durant la sécheresse 1969-1974, Travaux et Documents de Géographie Tropicale, $n^{\circ}$ 30. Bordeaux, CEGET-CNRS, p. 201-217.

BERNUS E., 1979 - « Le contrôle du milieu naturel et du troupeau par les éleveurs touaregs sahéliens ", in Pastoral production and society, Cambridge University Press/MSH, Paris/Cambridge, p. 67-74.

BERNUS E., 1981 - Touaregs nigériens. Unité culturelle et diversité régionale d'un peuple pasteur. Paris, ORSTOM, 507 p. (Réédition 1993, Paris, L'Harmattan).

BERNUS E., 1982 - «Territoires nomades. Approche d'un géographe ». Paris, Maison des Sciences de l'Homme, Production Pastorale et Société, $\mathrm{n}^{\circ}$ 11, p. 84-90.

BERNUS E., 1988 - « La représentation de l'espace chez les Touaregs du Sahel ». Montpellier, Mappemonde, $\mathrm{n}^{\circ}$ 3, 6 cartes, p. 1-5.

BERNUS E., 1993 - Touaregs nigériens. Unité culturelle et diversité régionale d'un peuple pasteur. Paris, L'Harmattan, 6 pl. photos, 30 fig., 5 cartes h. t, index, biblio. 507 p. ( $1^{\circ}$ édition Mém. ORSTOM $\mathrm{n}^{\circ}$ 94, 1981).

BERNUS E., 1999 - « Graines sauvages récoltées par les Touaregs sahéliens ». Encyclopédie Berbère, Aix-en-Provence, vol. XXI, p. 3199-3208.

BLANC-PAMARD Ch. \& BOUTRAIS J., 1994 - Dynamique des systèmes agraires, à la croisée des parcours pasteurs éleveurs cultivateurs, ORSTOM, $336 \mathrm{p}$.

BONTE P., 1973 - L'Élevage et le commerce du bétail dans l'Ader Doutchi-Majya, Niamey, CNRSH (Centre nigérien de recherche en sciences humaines).

BONTE P., 1971 - Production et échanges chez les Touareg Kel Gress du Niger, Paris, Institut d'Ethnologie.

BOURGEOT A., 1994 - «L'agro-pastoralisme des Touaregs Kel Ewey (Aïr, Niger) », in Au contact Sahara-Sahel, Milieux et Sociétés au Niger, Revue de géographie alpine, vol. 1, h.s., p. 137-156.

CAPOT-REY R., 1942 - « Le nomadisme pastoral dans le Sahara français », Trav. IRS, t. I, p. 63-86.

CLAUDOT-HAWAD H., 1986 - « La conquête du « vide » ou la nécessité d'être nomade », Revue de l'Occident Musulman et de la Méditerranée $\mathrm{n}^{\circ}$ 41-42, 397-412.

CLAUDOT-HAWAD H., 1990 - « Nomades et États : L'impensé juridique », Droit et Société, n 15, p. 229-242. 
CLAUDOT-HAWAD H., 1999 - «L'oubli du désert. Regard nomade sur la modernité territoriale », in Ethnies, Série Documents, $\mathrm{n}^{\circ} 24$, Paris.

CLAUDOT-HAWAD H., 2001 - Eperonner le monde. Nomadisme, cosmos et politique chez les Touaregs. Aixen-Provence, Edisud.

CLAUDOT-HAWAD H. (éd.), 2002a - Voyager d'un point de vue nomade, Paris, Paris-Méditerranée.

CLAUDOT-HAWAD H., 2002b - Touaregs, Apprivoiser le désert, Paris, Gallimard.

CLAUDOT-HAWAD H., 2005 - « Sahara et nomadisme, L'envers du décor », REMMM, n 111-112, p. 221-244.

CLAUDOT-HAWAD H., 2006 - « A Nomadic Fight against Immobility : the Tuareg in the Modern State ", in D. Chatty (ed.), Nomadic Societies in The Middle East and North Africa : Entering the 21st Century, Leiden, Brill, 654-681.

CLAUDOT-HAWAD H., 2008 - « Un territoire bâti comme une tente nomade ", Réfractions, $\mathrm{n}^{\circ}$ 21, p. $51-60$

CLAUDOT-HAWAD H., 2011 - "'Avoir de la cendre' : ancrages nomades en territoire touareg ", Techniques et cultures $\mathrm{n}^{\circ}$ 56, 2011/1, p. 78-97.

CLAUDOT-HAWAD H. \& HAWAD (éds.), 1993 - Voix solitaires sous l'horizon confisqué, Paris, Ethnies, 253 p. COLLOQUE 1966 - Deuxième colloque sur le nomadisme pastoral et la sédentarisation en Afrique et en Asie Paris, Paris, CHEAM, $33 \mathrm{p}$.

COLLOQUE 1973 - Pastoral production and society (Actes du colloque international sur le pastoralisme nomade), Paris, MSH, $493 \mathrm{p}$.

COPANS J. (dir.), 1975 - Sécheresses et famines au Sahel, Paris, Maspéro, 2 tomes, Dossiers africains, 155 et $143 \mathrm{p}$.

DeleUZe G. \& GUATTARI F., 1980 - Mille plateaux. Paris, Editions de Minuit.

DOCUMENTATION FRANÇAISE (La) 1953 - L'économie pastorale saharienne, Paris, La Documentation Française, $n^{\circ} 1730,21$, avril.

DOUTRESSOULLE G., 1952 - L'élevage au Soudan français, (2éd. augmentée), Alger, Imbert, 383 p. DU BoIS V. D., 1974 - « The drought in Niger. Part III : The flight of the Malian Tuareg - Part IV : the new refugee camp at Lazaret », Fieldstaff Reports. West African Studies, 15, n ${ }^{\circ} 6$ et 7, 21 p. GALLAIS J., 1975 - Pasteurs et paysans du Gourma. La condition sahélienne, Paris, CNRS, 240 p. GALLOY P., 1958 - Nomadisation et sédentarisation dans les cercles de Goundam et de Tombouctou (mission d'étude et d'aménagement du Niger), Bamako, Trav. Publ., 144 p.

GAST, M., 1968 - Alimentation des populations de l'Ahaggar, étude ethnographique, Paris, Arts et Métiers Graphiques, $457 \mathrm{p}$.

GAST M., 2000 - Moissons du désert. Utilisation des ressources naturelles en période de famine au Sahara central, Paris, Ibis Press (Illustré de photos et dessins, glossaire des noms de plantes et d'animaux en tamahaq.)

HINCKER C., 2004 - «Représentations sociales de la domestication des animaux sauvages dans l'ouest du monde touareg (Mali) », Anthropozoologica 39 (1), p. 275-288.

MARTY, A., 1975 - Contribution à la relance des coopératives d'éleveurs en 6e région du Mali. Rapport de mission, p. mars-mai, Paris, IRAM, 125 p. 
MARTY A., 1985 - Crise rurale en milieu nord-sahélien et recherche coopérative. L'expérience des régions de Gao et Tombouctou. Mali, 1975-1982., Thèse pour le doctorat d'Etat, sous la direction de G. Belloncle, Université François Rabelais-Tours, 2 tomes, 927 p.

MORTIMORE M. J., 1972 - "The changing ressources of sedentary communities in Air, Southern Sahara", The Geographical review, vol.62/1, p. 71-91.

NICOLAISEN J., 1963 - Ecology and culture of the pastoral Tuareg with particular reference to the Tuareg of Ahaggar and Ayr, Copenhague, Nat. Museum, 548 p.

NiCOLAISEN J. \& NICOLAISEN I., 1997 - The Pastoral Tuareg Ecology, Culture and Society, Copenhague, Thames \& Hudson, 2 vol.

NICOLAS F., 1947 - « La transhumance chez les Iulleminden de l'Est », Trav. IRS, 4, p. 111-126.

NORRIS H. T., 1952 - « Touareg nomadism in the modem world », Afr. Affairs (Londres), nº 51, p. $152-155$.

OULD SIDI MOHAMED Z., 1990 - Le développement et les conflits fonciers dans la boucle du Niger : la vallée du cercle de Gourma Rharous (Mali), Mémoire de DEA en Sociologie du Développement, sous la direction de E. Le Roy, Université de Paris I-Sorbonne, $125 \mathrm{p}$.

PEDERSON J \& BENJAMINSEN T. A,. 2008 - "One Leg or Two ? Food Security and Pastoralism in the Northern Sahel”, Human Ecology, vol. 36, Is. 1, February 2008, p. 43-57.

PIERRE M., 1906 - L'élevage en AOF, Paris, Challamel.

RANDALL S. \& GIUFFRIDA A., 2005 - « Forced migrations, Sedentarisation and Social Change : Malian Kel Tamasheq" in D. Chatty (ed.), Pastoralists of North Africa and the Middle East entering the 21 st Century, Leiden, Brill Publication, p. 431-463.

RETAILLE D., 1989 - « Le destin du pastoralisme nomade en Afrique », L'information géographique 53, p. 103-113.

ROGNON P., 1963 - « Problèmes des Touaregs du Hoggar », in UNESCO : Nomades et nomadisme au Sahara (recherches sur la zone aride), Paris, p. 59-66.

SIRCOULON J., 1985 - « La sécheresse en Afrique de l'Ouest. Comparaison des années 1982-84 avec les années 1972-73 », in Cahiers ORSTOM, série Hydrologie, vol. XXI, nº 4, p. 75-86.

SPITTLER G., 1993 - Les Touaregs face aux sécheresses et aux famines. Les Kel Ewey de l'Aïr (Niger), Paris, Karthala, 420 p.

SWIFT J., 1973 - « Le nomadisme pastoral en tant que forme d'utilisation des terres : les Touareg de l'Adrar des Ifoghas », Etudes Maliennes, 5, p. 35-44.

SWIFT J., 1981 - The economics of traditional nomadic pastoralism : the Twareg of the Adrar $n$ Iforas (Mali), Londres, University of Sussex, $394 \mathrm{p}$.

UNESCO, 1963 - Nomades et nomadisme au Sahara, Paris, UNESCO, 195 p.

VALLET M., 1960 - «Cycle de migration des tribus Hoggar dans l'Adrar des Ifoghas», Bull. de Liaison Sah., 11, $\mathrm{n}^{\circ} 40$, p. 347-351.

VALLET M., 1975 - « Les Touaregs et le développement », Options méditerranéennes, $\mathrm{n}^{\circ}$ 28, p. 34-41.

WALENTOWITZ S., 2003 - Enfant de Soi, enfant de l'Autre. La construction symbolique et sociale des identités à travers une étude anthropologique de la naissance chez les Touaregs (Kel Eghlal et Ayttawari de l'Azawagh, Niger), thèse de III ${ }^{\mathrm{e}}$ cycle, Paris, EHESS. 
WELET HALATINE F., 1996 - «L'abandon des privilèges. Parcours d'une femme touarègue dans la modernité », Ethnies, ${ }^{\circ}$ 20-21, p. 29-38.

\section{NOTES}

1. Voir à ce sujet Claudot-Hawad 1996.

\section{INDEX}

Mots-clés : Commerce, Economie, Ethnologie, Sahara, Touareg 